\title{
Psychosocial Burden Due to Acne Vulgaris Affects Treatment-Seeking Behavior in Medical Students in Jakarta, Indonesia
}

\author{
Franklind Matthew, ${ }^{1}$ Regina, ${ }^{2}$ Inneke Jane Hidajat, ${ }^{2}$ Melyawati ${ }^{2}$ \\ ${ }^{1}$ School of Medicine and Health Sciences, Atma Jaya Catholic University of Indonesia, \\ ${ }^{2}$ Department of Dermatology and Venereology School of Medicine and Health Sciences, Atma Jaya \\ Catholic University of Indonesia
}

\section{Abstract}

Background: Acne vulgaris (AV) is a chronic inflammation of the pilosebaceous unit of the skin that occurs in $85 \%$ of adolescents and can continue into adulthood. The skin of the facial area has an essential role in socializing, therefore, facial AV can contribute to the accumulation of psychosocial stress. The prevalence of individuals with AV seeking treatments is low in some countries. This study aimed to determine the association between psychosocial burdens due to AV and medical treatment-seeking behavior in young adults.

Methods: A cross-sectional study was conducted in February 2020 among 100 medical students with AV. The ASEAN Lehmann criteria were used to objectively assessed AV. Psychosocial burden was determined by the Cardiff Acne Disability Index (CADI) questionnaire and then classified into unaffected (score 0), mild (score 1-5), moderate (6-10), and severe (11-15). History-related AV was obtained through a questionnaire. The analysis was performed using the chi-square test.

Results: Of the 100 medical students with AV, $82 \%$ had suffered from mild to severe psychosocial burdens. The mean score of the CADI was 4.03 . However, only $44 \%$ of students sought treatment from doctors for their AV. There was a significant association between psychosocial burden and medical treatment-seeking behavior ( $\mathrm{p}=0.014)$.

Conclusions: The psychosocial burden of acne vulgaris in young adults is high, however, medical treatment-seeking behavior is low. Therefore, psychosocial management is an essential aspect in treating patients with AV.

Keywords: Acne vulgaris, CADI, psychosocial burden, treatment-seeking behavior

\section{Introduction}

Acne vulgaris (AV) is a chronic inflammation of the pilosebaceous unit that occurrs in $85 \%$ of adolescents and can continue into adulthood. ${ }^{1}$ The prevalence of $\mathrm{AV}$ in the United States $^{2}$ is $85 \%$ in the age group $12-24$ years, mainly due to hormonal changes. Many factors affect the $\mathrm{AV}$ prevalence, such as race, age, and gender. At the age of 18-22 years, more men were found to experience AV than women, but starting at the age of 23 years, the prevalence of $A V$ in the female group was considerably higher. Some typical AV skin lesions include blackheads, papules, pustules, nodules, and cysts. Severe AV conditions can cause scarring and psychosocial stress. ${ }^{3}$

The skin, especially the face, has a vital role in socializing. Acne can cause disturbances in social functioning, such as decreased self-confidence, academic performance, and contribute to psychological burdens, especially in adolescents and young adults. Associated conditions include increased anxiety, depression, and frustration, which in turn affects their quality of life. ${ }^{4,5}$ Although $\mathrm{AV}$ affect increased of psychosocial burden, several studies reported that only less than $20 \%$ of adolescents with $\mathrm{AV}$ in the United States and less than $51 \%$ of young adults in Jordan sought treatment from medical doctors. ${ }^{3,6}$ Many cases have experienced AV

Correspondence: dr. Regina, Sp.KK, Department of Dermatology and Venereology, School of Medicine and Health Sciences, Atma Jaya Catholic University of Indonesia-Atma Jaya Hospital, Jalan Pluit Raya No. 2 North Jakarta, Indonesia, Email: regina@atmajaya.ac.id 
for years before seeking treatments. Some of the reasons are embarrassment, unsuitable schedules, or self-medication with over the counter (OTC) products. Many OTC products for $\mathrm{AV}$ treatment are widely available due to lower cost, convenience, and increasing usage trend in society. ${ }^{6}$

The proportion of the young adults in Indonesia who seek AV treatment from doctors, either a dermatologist or a general practitioner, is not widely known. This study was conducted to determine the severity of $\mathrm{AV}$, the psychosocial burden it incurred, and the relationship between this burden and treatment-seeking behavior among young adults.

\section{Methods}

A cross-sectional study was conducted in February 2020, conducted at the School of Medicine and Health Sciences, Atma Jaya Catholic University of Indonesia. The minimum sample size was 61 respondents considering the $80 \% \mathrm{AV}$ prevalence, a confidence level of $95 \%$, and a degree of deviation of $10 \%$. The inclusion criteria for this study were medical students who suffered from acne vulgaris and willing to participate in this study by signing an informed consent. Exclusion criteria were students with hormonal disorders such as Polycystic Ovarian Syndrome (PCOS), Cushing's syndrome, and others.

The questionnaire was distributed and filled out by each respondent, consisting of three parts. The first part contained demographical data, and the second part consisted of questions regarding acne treatment behavior. The third part referred to the psychosocial burden due to $\mathrm{AV}$, which was assessed using the Cardiff Acne Disability Index (CADI) questionnaire. The classification of the psychosocial burden was divided into unaffected (score 0 ), mild (score $1-5)$, moderate (6-10), and severe (11-15).
Then, the dermatologist assessed the AV degree according to the ASEAN Lehmann classification; mild designated as $<20$ comedones, $<15$ papules or pustules, without nodule; moderate for 20-100 comedones, 1550 papules or pustules, $<5$ nodules; and severe for $>100$ comedones, $>50$ papules or pustules, $>5$ nodules.

The ethical clearance was obtained from the ethics committee of the School of Medicine and Health Sciences, Atma Jaya Catholic University of Indonesia with the number: 12/01/KEPFKUAJ/2020.

Univariate data analysis used descriptive statistics. Bivariate analysis was performed using the Chi-square test to determine the relationship between the psychosocial burden of $\mathrm{AV}$ and medical treatment-seeking behavior. The relationship was declared significant if the $p$-value is $<0.05$. Statistical analysis using the SPSS version 25.0.

\section{Results}

Demographic characteristic showed that out of 100 study participants, there were more women $(65 \%)$ than men, with an age range of $19-22$ years old and a mean age of $20.3+0.77$ years (Table 1 ).

Most of the students suffered from AV for about 5 to 9 years (57\%), with a period of suffering of at least $\geq 10$ years (3\%). The degree of AV severity on the face was assessed by dermatologists using the ASEAN Lehmann classification with most students had a mild classification $(74 \%)$ and the least students suffering from severe AV (3\%). Interestingly, only $44 \%$ of the students went to a medical doctor, either a general practitioner or a dermatologist, to treat their $\mathrm{AV}$. Based on currently used AV therapies, $59 \%$ of students used OTC products, $4 \%$ used medications prescribed by doctors, and the rest combined both categories (Table 2).

Table 1 Demographic Characteristic of Study Participants $(n=100)$

\begin{tabular}{lcc}
\hline Demographic Characteristic & $\mathbf{n}$ & $\mathbf{\%}$ \\
\hline Age (years) & & \\
19 & 8 & 8 \\
20 & 54 & 54 \\
21 & 30 & 30 \\
22 & 8 & 8 \\
Gender & & \\
Men & 35 & 35 \\
Women & 65 & 65 \\
\hline
\end{tabular}


Table 2 Characteristic of Acne Vulgaris among Students Aged 19-22 years

\begin{tabular}{lcc}
\hline \multicolumn{1}{c}{ AV-related data } & $\mathbf{n}$ & $\mathbf{\%}$ \\
\hline Duration of suffering from AV & & \\
$\quad$ 5 years & 40 & 40 \\
$5-9$ years & 57 & 57 \\
$\quad$ 10 years & 3 & 3 \\
AV Degree & 74 & 74 \\
$\quad$ Mild & 23 & 23 \\
$\quad$ Moderate & 3 & 3 \\
$\quad$ Severe & & \\
Treatment to doctor & 44 & 44 \\
$\quad$ Yes & 56 & 56 \\
$\quad$ No & & \\
Current medication used & 4 & 4 \\
Medications from doctor & 56 & 59 \\
OTC products & 40 & 37 \\
Combination & 100 & 100 \\
Total & & \\
\hline
\end{tabular}

Note: $\mathrm{AV}=$ Acne vulgaris, $\mathrm{OTC}=$ Over the counter

Several subjects used other OTC products (Table 3), with the most widely used OTC products for treating $\mathrm{AV}$ was facial soap $(n=89)$, followed by acne spot drugs $(n=52)$, and sheet masks $(\mathrm{n}=46)$.

Furthermore, data on the psychosocial burden of $\mathrm{AV}$ and medical treatment-seeking behavior clarified two points. First, students who had a psychosocial burden due to AV suffered the most most mild burden $(60 \%)$ and severe burden (9\%). The mean value of psychosocial burden obtained based on the CADI score was 4.03. Second, there was a relationship between the psychosocial burden due to $\mathrm{AV}$ and medical treatment-seeking behavior $(\mathrm{p}=0.014)$ (Table 4).

\section{Discussion}

This study found that most (82\%) students aged 19-22 years old suffered from mild to severe psychosocial burdens due to acne vulgaris, with a mean CADI score of 4.03. There were $44 \%$ of students who sought treatment to a doctor for their AV. There is a statistically significant relationship between psychosocial burden and medical treatmentseeking behavior $(\mathrm{p}=0.014)$.

Most of the students (57\%) had suffered from $A V$ for 5-9 years. This result is similar to a previous study in Europe which showed that the mean $\mathrm{AV}$ onset has occurred at the age of $14 \pm 1.9$ years (range $10-24$ years). ${ }^{9}$ The

Table 3 Over the Counter Products Used by Students

\begin{tabular}{|c|c|}
\hline OTC Products & $\mathbf{n}$ \\
\hline Facial soap & 89 \\
\hline Acne spot drugs & 52 \\
\hline Sheet masks & 46 \\
\hline Night creams & 40 \\
\hline Serums & 32 \\
\hline Face masks & 32 \\
\hline Cleansing oils & 29 \\
\hline Scrubs & 26 \\
\hline Others (moisturizer, toner, etc.) & 17 \\
\hline
\end{tabular}


Table 4 Association between Psychosocial Burden Due to Acne Vulgaris and Medical Treatment-seeking Behavior among Students Aged 19-22 years old

\begin{tabular}{llcccccc}
\hline & & \multicolumn{4}{c}{ Treatment from Doctor } \\
\cline { 3 - 7 } & & Total & \multicolumn{2}{c}{ Yes } & \multicolumn{2}{c}{ No } & P-value \\
\cline { 3 - 7 } & & & $\mathbf{n}$ & $\mathbf{0}$ & $\mathbf{n}$ & $\mathbf{0}$ & \\
\hline \multirow{2}{*}{ Psychosocial burden } & Unaffected & 13 & 5 & 11.4 & 8 & 14.3 & 0.014 \\
& Mild & 60 & 20 & 45.4 & 40 & 71.4 & \\
& Moderate & 18 & 13 & 29.5 & 5 & 8.9 \\
& Severe & 9 & 6 & 13.7 & 3 & 5.4 \\
\hline
\end{tabular}

occurrence of $\mathrm{AV}$ in adolescence is associated with increased sebum production in the pilosebaceous unit related to androgenous hormones. Although it affects about 85\% of adolescents, AV can also occur before or after adolescence due to its multifactorial pathogenesis. ${ }^{10}$

Most (74\%) students had mild AV, and only $3 \%$ had severe AV, similar to another study showing about $70 \%$ mild $A$. $^{1}$ In choosing AV therapy, only half of the students (44\%) went to a doctor to treat their AV. The rate of visits to the doctor due to $\mathrm{AV}$ is low, some of which are the feeling that the AV condition is not too severe, busy schedule, and shyness to consult. ${ }^{11}$ Another similar study in Pakistan also showed about $55 \%$ of students seeking help from a doctor for their AV problems. ${ }^{12}$ However, this study did not investigate why the study subjects did not consult their $\mathrm{AV}$ problems with their doctor.

Self-medication with OTC products was found in $56 \%$ of students, and $44 \%$ had used a combination of prescription drugs with OTC products. Some of the reasons for selfmedication include mild $\mathrm{AV}$ degrees, available OTC products, lack of time to see a doctor, and knowledge of pharmacology as a medical student. ${ }^{12}$ Sources of information related to $\mathrm{AV}$ treatment were mostly obtained through friends or seniors in medical school and family members who are doctors. These results are similar to several previous studies in Jordania ${ }^{3}$ and Saudi Arabia. ${ }^{6}$ The most widely used OTC products by students include facial soaps and topical therapies, such as acne spots, night creams, masks, and serums.

The severity of the psychosocial burden due to AV was mostly mild, with an average CADI score of 4.03. Several other studies have also revealed similar results. ${ }^{11,13}$ Thus, there is thus a significant relationship between psychosocial burden due to AV and medical treatmentseeking behavior $(p<0.05)$, although another study showed a different result. ${ }^{11}$

Interestingly, a study among 852 French adolescents found that nearly two-thirds wanted more information about acne and $38.6 \%$ had never consulted a physician. More than half of the respondents felt ashamed of their skin condition and almost a third felt that AV interfered with their social and leisure activities. With society becoming increasingly beauty-conscious, there may be additional pressure on young adults to achieve "flawless" skin. As expected, the more severe the acne degree, the more likely a person is to seek medical treatment. ${ }^{14}$ Moreover, 58.9\% of participants who had received medical treatment would rate their acne as "excellent", "very good" or "good" compared to up to $41.3 \%$ of participants seeking non-medical treatment. $^{15}$

Acne has been known to have a significant impact on psychological and social life. The CADI questionnaire used in this study is a specific questionnaire for $\mathrm{AV}$ sufferers describing feelings of shame, frustration, aggression, disturbances in social life, or relationships with the opposite gender, public avoidance behavior, concerns about skin appearance, and self-perception problems about $A V^{5}$ Our results indicate that there is a psychosocial burden due to acne, therefore, psychosocial factors also need to be considered when treating AV patients.

The strength of this study is the use of a special CADI questionnaire to assess the psychosocial burden of AV. Moreover, an objective assessment of the degree of acne has been performed by a dermatologist. The weakness of this study is that there is no further exploration of why the participants choose to seek a doctor or not.

In conclusion, this study concludes that there is a significant association between psychosocial burdens caused by acne vulgaris and medical treatment-seeking behavior. 
These results are expected to assist doctors in carrying out AV management holistically by considering the psychological factors of the patients.

\section{References}

1. Zari S, Turkistani A. Acne vulgaris in Jeddah medical students: prevalence, severity, selfreport, and treatment practices. Journal of Cosmetics, Dermatological Sciences and Application. 2017;7(1):67-76.

2. Raikar DR, Manthale NS. A cross sectional study of self-medication for acne among undergraduate medical students. Int J Res Dermatol. 2018. 25;4(2):211-4.

3. Jaber RM, Alnshash BM, Mousa SN, Fayoumi HS, Al-Qaderi LM, Zant AM. The epidemiology of acne vulgaris among adolescents and young adults in Jordan University Hospital. Open Journal of Nursing. 2020;10(4):353-66.

4. Hosthota A, Bondade S, Basavaraja V. Impact of acne vulgaris on quality of life and self-esteem. Cutis. 2016;98(2):121-4.

5. Kumar S, Singh R, Kaur S, Mahajan BB. Psychosocial impact of acne on quality of life in North India: a hospitalbased cross-sectional study. Journal of Pakistan Association of Dermatologists. 2016;26(1):35-9.

6. Alshehri M, Almutairi A, Alomran A, Alrashed B, Kaliyadan F. Over-the-counter and prescription medications for acne: A cross-sectional survey in a sample of university students in Saudi Arabia. Indian Dermatol Online J. 2017;8(2):120-3.

7. Abdelrazik YT, Ali FM, Salek MS, Finlay AY. Clinical experience and psychometric properties of the Cardiff Acne Disability Index (CADI). $\mathrm{Br}$ J Dermatol. 2021. doi:10.1111/bjd.20391.

8. Tayel K, Attia M, Agamia N, Fadl N. Acne vulgaris: prevalence, severity, and impact on quality of life and self-esteem among Egyptian adolescents. J Egypt Public Health Assoc. 2020;95(1):30.

9. Szepietowski JC, Wolkenstein P, Veraldi S, Tennstedt D, Machovcová A, Delarue A. Acne across Europe: an online survey on perceptions and management of acne. J Eur Acad Dermatol Venereol. 2018;32(3):4636.

10. Picardo M, Eichenfield LF, Tan J. Acne and rosacea. Dermatol Ther (Heidelb). 2017;7(Suppl 1):43-52.

11. Desai KP, Martyn-Simmons C, Viner $\mathrm{R}$, Segal T. Help-seeking behaviours, opportunistic treatment and psychological implications of adolescent acne: crosssectional studies in schools and hospital outpatient departments in the UK. BMJ Open. 2017;7(9):e016964.

12. Tameez-ud-din A, Malik IJ, Bhatti AA, TameezUd Din A, Sadiq A, Khan MT, et al. Assessment of knowledge, attitude, and practices regarding self-medication for acne among medical students. Cureus. 2019;11(8): e5510.

13. Bajawi S, Salih S, Mahfouz MS, Bajawi $\mathrm{N}$, Asiri B. Acne vulgaris awareness and impact on quality of life and psychological status of adolescent school children in Jazan, Saudi Arabia. International Journal of Sciences: Basic and Applied Research. 2016;25(2):374-84.

14. de Vries F, Tjin E, Driessen R, Vehof H, van de Kerkhof P. Exploring patient journeys through acne healthcare: a patient perspective. J Dermatolog Treat. 2021;1-8. doi: 10.1080/09546634.2021.1940808

15. Su P, Chen Wee Aw D, Lee SH, Han Sim Toh MP. Beliefs, perceptions and psychosocial impact of acne amongst Singaporean students in tertiary institutions. J Deutsch Dermatol Ges. 2015;13(3):227-33. 